\title{
Parametric analyses of attack-fault trees
}

\author{
Étienne André \\ Université Paris 13, LIPN, CNRS, F-93430, Villetaneuse, France \\ JFLI, CNRS, Tokyo, Japan / National Institute of Informatics, Tokyo, Japan
}

\author{
Didier Lime \\ École Centrale de Nantes, LS2N, CNRS, \\ UMR 6004, Nantes, France
}

\author{
Mathias Ramparison \\ Université Paris 13, LIPN, CNRS, \\ UMR 7030, F-93430 Villetaneuse, France
}

\author{
Mariëlle Stoelinga \\ Formal Methods and Tools, University of Twente, \\ The Netherlands
}

\begin{abstract}
Risk assessment of cyber-physical systems, such as power plants, connected devices and IT-infrastructures has always been challenging: safety (i. e., absence of unintentional failures) and security (i. e., no disruptions due to attackers) are conditions that must be guaranteed. One of the traditional tools used to help considering these problems is attack trees, a treebased formalism inspired by fault trees, a well-known formalism used in safety engineering. In this paper we define and implement the translation of attack-fault trees (AFTs) to a new extension of timed automata, called parametric weighted timed automata. This allows us to parametrize constants such as time and discrete costs in an AFT and then, using the model-checker IMITATOR, to compute the set of parameter values such that a successful attack is possible. Using the different sets of parameter values computed, different attack and fault scenarios can be deduced depending on the budget, time or computation power of the attacker, providing helpful data to select the most efficient counter-measure.

Index Terms-security, attack-fault trees, parametric timed automata, IMITATOR
\end{abstract}

\section{INTRODUCTION}

In the past few years, the range of security breaches in the security of organizations has become larger and larger. The process of unifying them by determining relations and consequences between separated events has become more difficult: how to relate the presence of solid oxygen in a helium tank in SpaceX rocket Falcon 9 to its the explosion during firing tests? What is the cost for the attacker and the damages caused to SpaceX manufacturing plants? One of the tools available to help structure risk assessments and security analyses is attack trees, recommended, e. g., by NATO Research and Technology Organisation (RTO) [1] and OWASP (Open Web Application Security Project) [2]. Attack trees [3] were formalized in [4] as a popular and convenient formalism for security analysis (see [5] for a survey) and are inspired by fault trees [6], [7] a well-known formalism used in safety engineering. Bottom-up computation for a single parameter (e. g., cost, probability or time of an attack), can be performed directly on attack trees [8]. Attack trees and fault trees are quite similar but differ on their gates and/or goals [8], [9]. Both

This work is partially supported by the ANR national research program PACS (ANR-14-CE28-0002), the PHC Van Gogh project PAMPAS, by STW under the project 15474 SEQUOIA, KIA KIEM project 628.010.006 StepUp, the EU under the project 102112 SUCCESS and ERATO HASUO Metamathematics for Systems Design Project (No. JPMJER1603), JST. are constructed with leaves that model component and attack step failures or successes that propagate through the system via gates. While fault trees focus on safety properties, attack trees considerate skills, resources and risk appetite possessed by an attacker performing actions. Attack-fault trees (AFTs) [10] combine safety properties from fault trees and security conditions from attack trees; therefore gates of both fault trees and attack trees are used in this formalism.

Quantitative analysis of AFTs with multiple quantitative annotations on AFTs like cost, time, failure probabilitieswhich can functionally be dependent on each other-evaluates risks and helps to determine the most risky scenarios and therefore to select the most effective counter-measures.

a) Contribution: In this work, we study a more abstract version of the security problem, and we propose an approach to synthesize times and costs necessary to individual actions in order to perform a successful attack or individual failures causing the failure of the entire system. The global attack time and cost can then be expressed as a combination of the parametric unit costs. To this end, we propose a formalization of attack-fault trees using an ad-hoc extension of parametric timed automata called parametric weighted timed automata (PWTAs). PWTAs can be seen as a generalization of parametric timed automata (PTAs) [11] and weighted/priced automata [12], [13] with only costs on transitions.

We implement our framework within the tool ATTop presented in [14], allowing to define AFTs in the Galileo format, and provide an automated translation into the IMITATOR input format [15].

As a proof of concept, we apply our framework to an attack tree of [10] and an original attack-fault tree. With the help of the parametric timed model checker IMITATOR, we are able to synthesize constraints in several dimensions; further we discuss induced possible attack and fault scenarios.

This enlarges the scope of quantitative analysis for AFTs by parameterizing multiple annotations on the AFT at once such as time, cost and damages and then compute for instance the optimal combination of parameter values for the attack to fail quickly while keeping damages to the system low.

b) Related work: Attack tree analysis has been studied through lattice theory [4], timed automata [16], [10], [14], I/O-IMCs [17], [18], Bayesian networks [19], Petri nets [20], 
stochastic games [21], [22], etc. Uppaal has been used for model transformations in [23] and in [24] UML sequence diagrams are manually transformed into timed automata models. [10] especially tackles the problem of multiple complex risk metrics and attacker profiles, in a probabilistic and timed formalism that can be computed and analyzed using stochastical model-checking [25] and Uppaal SMC [26]. AFTs are modeled in the Galileo format and translated with the tool ATTop [14] into stochastic timed automata [27].

However, synthesis of multidimensional parameters (time, cost for the attacker, damages for the organization...) at once for fully timed systems is not treated in the previously cited works, and these works require testing one by one a set of possible attribute values for an AFT.

Besides, attack-defense trees are one of the most wellstudied extensions of attack trees and new analysis methods are still developed [9], [28].

In a completely different area, asynchronous hardware circuits' gates were translated into (parametric) timed automata in [29]; our translation of AFTs gates into PWTAs synchronized using parallel composition shares some similarities with that approach.

c) Outline: We recall attack-fault trees in Section II. We then introduce the formalism of parametric weighted timed automata in Section III. Our translation from AFTs to PWTAs is given in Section IV. Then, we describe our implementation in Section V and report on experiments in Section VI. We conclude by discussing future works.

\section{Attack-Fault Trees}

Attack-fault trees (AFTs) model how a safety or security goal can be refined into smaller sub-goals, represented as gates, until no further refinement is possible, represented as leaves. The leaves of the tree model are either basic component failures (BCF) or basic attack steps (BAS). Since subtrees can be shared in the literature (see e.g., [10]), AFTs are actually directed acyclic graphs, rather than trees. In this paper, we consider only trees without shared gates or leaves. Safety is compromised with the failure of a BCF, i. e., without any outside spark action. Security is compromised when an outside attacker causes the activation of a BAS. Following the terminology of [10], in this paper write that a gate or a leaf is disrupted if the output is true i.e., it succeeds, and fails otherwise. A success event (disruption) models the fact that a component (gate or leaf) is compromised i.e., the attack is successful or the component fails. In contrast, a fail event models the robustness of the component against an attacker through a BAS, or a BCF.

\section{A. AFT leaves}

AFT leaves are equipped with an execution time and a rich cost structure that includes the cost incurred by an attacker and the damage inflicted on the organization. In contrast to [7], [10] where BCF and BAS are equipped with probability distributions, we consider both $\mathrm{BCF}$ and $\mathrm{BAS}$ as parametric time-dependent events. This allows us to compute a range

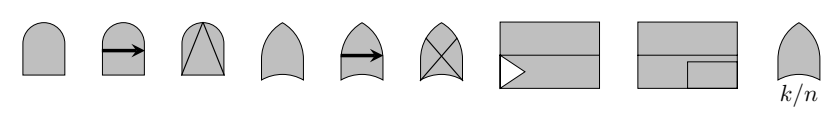

Figure 1: From left to right: AND, SAND, PAND, OR, SOR, XOR, FDEP, SPARE, VOT $(k / n)$ gates

of cost values, damages values and time intervals at once in order to perform operations such as optimum time values for a counter-measure while keeping damage to the organization low, and cost for the attacker high.

\section{B. AFT gates}

In order to model complex scenarios with multiple leaves, $\mathrm{BCF}$ and BAS have to be composed. For this purpose, logical gates are used that output either the propagation of a disruption, or not. Gates take as an input either leaves or outputs from gates in their subtrees. Logical gates used in AFTs are taken from both dynamic fault trees and attack trees: AND, PAND, SAND, OR, SOR, FDEP, SPARE, VOT $(k / n)$, depicted in Fig. 1. These gates are the translatable ones in ATTop [14] from the Galileo format. We also added the XOR gate to improve our modeling capabilities.

AND gate propagates a disruption (i.e.,, it synchronizes a success event [10]) if all of its children are disrupted, regardless of the order of disruption. Children are activated initially by the AND. Children of a SAND gate are activated sequentially from left to right. After the success (disruption) of the leftmost child, the second left most child is activated, and so on until the disruption of rightmost child. If all children are disrupted, the SAND gate is disrupted. However, if any child fails (to be disrupted), the SAND gate directly fails. SAND gate is a specific gate of attack trees. Compared with SAND gate, all children of a PAND gate are activated initially when the PAND gate is activated. The rest of the execution is similar to a SAND gate, and propagates a disruption if all children are disrupted from left to right (which in contrast is not mandatory for an AND gate), otherwise the PAND gate fails.

OR gate propagates a disruption if at least one of its children is disrupted. Children are activated initially by the OR gate. Similarly to a SAND gate, children of a SOR gate are activated sequentially after the termination of the previous one and from left to right. It propagates a disruption when one of its children is disrupted, otherwise if all children fail the SOR gate fails. XOR gate propagates a disruption if one of its children is disrupted and the other one fails.

FDEP (functional dependency) gate consists of a trigger event and several dependent events, and is a specific gate of fault trees. When the trigger event occurs, all its dependent $\mathrm{BCF}$ events are disrupted (i.e., the failure of the power supply automatically deactivate the alarm and security cameras, therefore the BCFs are successful).

SPARE gate is similar to SAND, but is a specific gate for fault events while SAND gate is used for attack events. SPARE gate consists of one primary BCF and several secondary $\mathrm{BCF}$ which are activated sequentially. If the primary 
BCF is disrupted (i.e., the component fails), a secondary becomes primary. If no BCFs are left (they all are disrupted), SPARE gate propagates a disruption.

$\operatorname{VOT}(k / n)$ gate is similar to OR gate and consists of $n \in \mathbb{N}$ children initially activated. $\operatorname{VOT}(k / n)$ gate is disrupted when $k$ of its $n$ children are disrupted.

\section{PARAmetric Weighted TIMED AUtomata}

Let $\mathbb{N}, \mathbb{Q}, \mathbb{Q}_{+}$, and $\mathbb{R}_{+}$denote the set of non-negative integers, rationals, non-negative rationals and non-negative reals, respectively.

We assume a set $\mathbb{C}=\left\{x_{1}, \ldots, x_{H}\right\}$ of clocks, i. e., realvalued variables that evolve at the same rate. A clock valuation is a function $\nu: \mathbb{C} \rightarrow \mathbb{R}_{+}$. We write $\overrightarrow{0}_{\mathbb{C}}$ for the clock valuation assigning 0 to all clocks. Given $d \in \mathbb{R}_{+}, \nu+d$ is s.t. $(\nu+$ $d)(x)=\nu(x)+d$, for all $x \in \mathbb{C}$. Given $R \subseteq \mathbb{C}$, we define the reset of a valuation $\nu$, denoted by $[\nu]_{R}$, as follows: $[\nu]_{R}(x)=$ 0 if $x \in R$, and $[\nu]_{R}(x)=\nu(x)$ otherwise.

We assume a set $\mathbb{T P}=\left\{p_{1}, \ldots, p_{J}\right\}$ of timing parameters. A timing parameter valuation $t v$ is $t v: \mathbb{T P} \rightarrow \mathbb{Q}_{+}$. We assume $\bowtie \in\{<, \leq,=, \geq,>\}$. A guard $g$ is a constraint over $\mathbb{C} \cup \mathbb{T P}$ defined by a conjunction of inequalities of the form $x \bowtie d$, or $x \bowtie p$ with $x \in \mathbb{C}, d \in \mathbb{N}$ and $p \in \mathbb{T P}$. Given $g$, we write $\nu \models t v(g)$ if the expression obtained by replacing each $x$ with $\nu(x)$ and each $p$ with $t v(p)$ in $g$ evaluates to true.

We assume a set $\mathbb{W}=\left\{w_{1}, \ldots, w_{M}\right\}$ of weights. A weight valuation $\mu$ is $\mu: \mathbb{W} \rightarrow \mathbb{Q}$. We write $\overrightarrow{0}_{\mathbb{W}}$ for the weight valuation assigning 0 to all weights. We assume a set $\mathbb{W P}=$ $\left\{q_{1}, \ldots, q_{N}\right\}$ of weight parameters. A weight parameter valuation $w v$ is $w v: \mathbb{W P} \rightarrow \mathbb{Q} .^{1}$ A linear arithmetic expression over $\mathbb{W} \cup \mathbb{W} \mathbb{P}$ is $\sum_{i} a_{i} w_{i}+\sum_{j} b_{j} q_{j}+c$, where $w_{i} \in \mathbb{W}$, $q_{j} \in \mathbb{W} \mathbb{P}$ and $a_{i}, b_{j}, c \in \mathbb{Q}$. Let $\mathcal{L} \mathcal{A}(\mathbb{W} \cup \mathbb{W P})$ denote the set of arithmetic expressions over $\mathbb{W}$ and $\mathbb{W} \mathbb{P}$. A parametric weight update is a partial function $\alpha: \mathbb{W} \nrightarrow \mathcal{L} \mathcal{A}(\mathbb{W} \cup \mathbb{W P})$. That is, we can assign a weight to an arithmetic expression of parametric weights and other weight values, and rational constants. Given a weight valuation $\mu$, a parametric weight update $\alpha$ and a weight parameter valuation $w v$, we need an evaluation function $\operatorname{eval}_{w v}(\alpha, \mu)$ returning a weight valuation, and defined as follows:

$$
\text { eval }_{w v}(\alpha, \mu)(w)= \begin{cases}\mu(w) & \text { if } \alpha(w) \text { is undefined } \\ \mu(w v(\alpha(w))) & \text { otherwise }\end{cases}
$$

where $\mu(w v(\alpha(w)))$ denotes the replacement within the linear arithmetic expression $\alpha(w)$ of all occurrences of a weight parameter $q_{i}$ by $w v\left(q_{i}\right)$, and of a weight variable $w_{j}$ with its current value $\mu\left(w_{j}\right)$. Observe that this replacement gives a rational constant, therefore $\operatorname{eval}_{w v}(\alpha, \mu)$ is indeed a weight valuation $\mathbb{W} \rightarrow \mathbb{Q}$. That is, eval ${ }_{w v}(\alpha, \mu)$ computes the new (non-parametric) weight valuation obtained after applying to $\mu$ the partial function $\alpha$ valuated with $w v$.

Parametric timed automata (PTA) extend timed automata [30] with timing parameters [11]. We extend further

\footnotetext{
${ }^{1}$ Observe that, in contrast to timing parameters that should be non-negative (which is usual for parametric timed automata), our weight parameters may be negative.
}

PTA with (discrete) rational-valued weight parameters, giving birth to parametric weighted timed automata (PWTA).

Definition 1. A parametric weighted timed automaton (PWTA) $\mathcal{A}$ is a tuple $\mathcal{A}=\left(\Sigma, L, l_{0}, F, \mathbb{C}, \mathbb{T P}, \mathbb{W}, \mathbb{W} \mathbb{P}, I, E\right)$ :

1) $\Sigma$ is a finite set of synchronization actions,

2) $L$ is a finite set of locations, $l_{0} \in L$ is the initial location,

3) $F \subseteq L$ is the set of accepting locations,

4) $\mathbb{C}$ is a finite set of clocks,

5) $\mathbb{T P}$ is a finite set of timing parameters,

6) $\mathbb{W}$ is a finite set of weights,

7) $\mathbb{W P}$ is a finite set of weight parameters,

8) $I$ is the invariant, assigning to every $l \in L$ a guard $I(l)$,

9) $E$ is a finite set of edges $e=\left(l, g, a, R, \alpha, l^{\prime}\right)$ where $l, l^{\prime} \in$ $L$ are the source and target locations, $g$ is a guard, $a \in \Sigma$, $R \subseteq \mathbb{C}$ is a set of clocks to be reset, and $\alpha: \mathbb{W} \nrightarrow$ $\mathcal{L} \mathcal{A}(\mathbb{W} \cup \mathbb{W} P)$ is a parametric weight update.

Given a timing parameter valuation $t v$ and a weight parameter valuation $w v$, we denote by $t v \mid w v(\mathcal{A})$ the nonparametric structure where all occurrences of a timing parameter $p_{i}$ have been replaced by $t v\left(p_{i}\right)$, and all occurrences of a weight parameter $q_{j}$ have been replaced by $w v\left(q_{j}\right)$. The resulting structure can be seen as an extension of a parametric weighted/priced timed automaton [12], [13] with only rational weights on edges. ${ }^{2}$ However, our structure goes beyond a simple parametric extensions of weighted/priced timed automata, for two reasons: $i$ ) we allow multiple weights; ii) we allow to not only increment weight values over a path, but also perform more complex operations on that weight, notably incrementing it with another weight value, which is clearly not possible in [12], [13]. Note that, if we restrict our parametric weight update function to expressions of the form $\alpha\left(w_{i}\right)=w_{i}+z$, where $z$ is either a weight parameter or a rational constant, then our formalism is exactly the parametric extension of (the discrete "switch" weight part of) [12], [13]. ${ }^{3}$

In addition, our formalism shares some similarities with the statically parametric timed automata of [31], where timed automata are extended with parameters that can only be used in guards, but not compared to clocks. In contrast, our weight parameters can only be used in updates, and not in guards; in addition, we also feature the timing parameters of [11] that can be compared to clocks.

Example 1. In the PWTA in Fig. 3, we have the following elements: $L=\left\{l_{1}, l_{2}, l_{3}\right\}, l_{0}=l_{1}$ (also the unique element of $F), \mathbb{C}=\{\mathrm{x}, \mathrm{y}\}$, and $\Sigma=\{$ press, prepare, serve $\}$, with the set $\mathbb{T P}=\left\{p_{1}, p_{2}\right\}$ and weights $\mathbb{W}=\{w\}, \mathbb{W P}=\{q\}$. There are four edges:

- $e_{1}=\left\langle l_{1}, g, a, R, l_{2}\right\rangle$ where $R$ sets both $\mathrm{x}, \mathrm{y}$ to $0, \alpha$ is $w=2 €$,

${ }^{2}$ In [13] cost is defined as the sum of each discrete cost on transitions (switch cost) plus the time spent in a location multiplied by an integer rate (duration cost), resulting in a rational value. Here, we omit the duration costs.

${ }^{3}$ Technically, as weighted/priced timed automata use integer constants, a rescaling of the constants is necessary: by multiplying all constants in $t v \mid w v(\mathcal{A})$ by the least common multiple of their denominators, we obtain an equivalent (integer-valued) weighted/priced timed automata. 


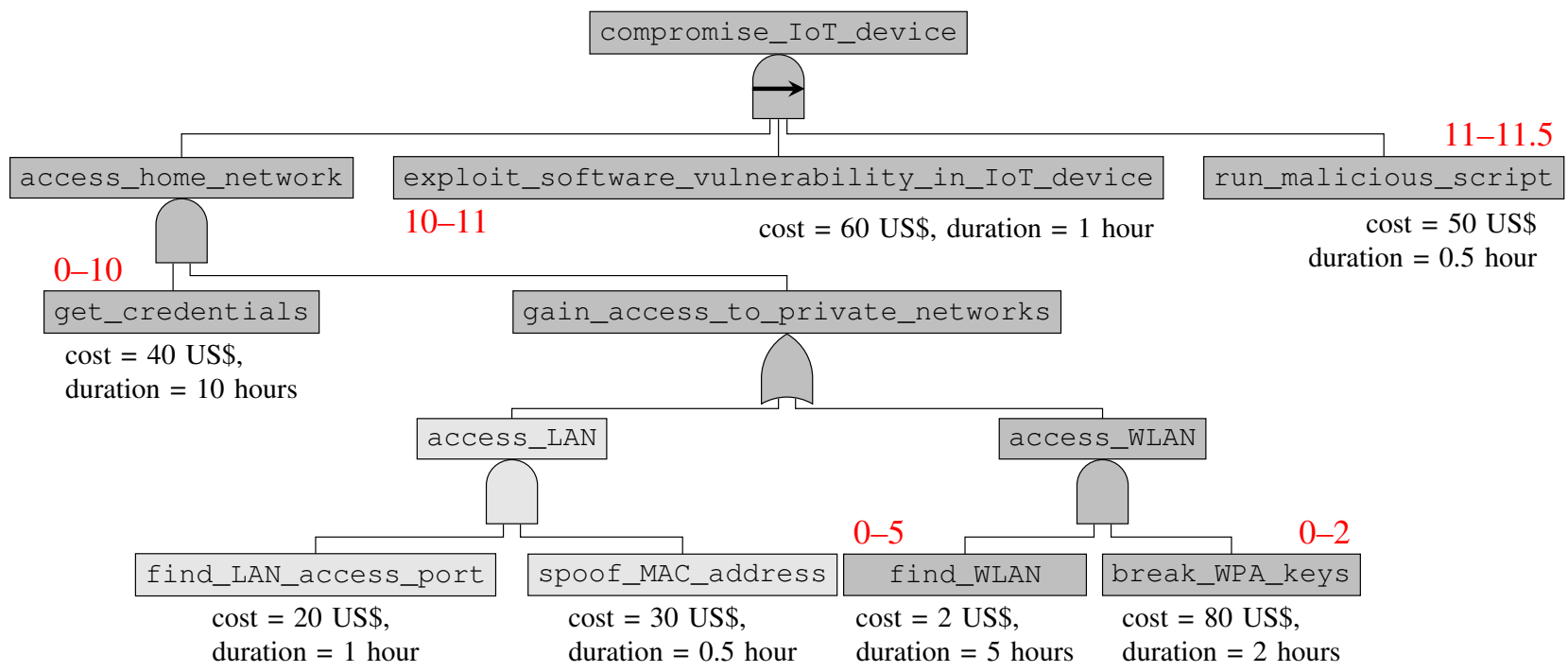

Figure 2: Attack Tree modeling the compromise of an IoT device from [23]. Leaves are equipped with the cost and time required to execute the corresponding step. The parts of the tree attacked in a successful attack are indicated by a darker color, with start and end times for the steps in this attack denoted in red.

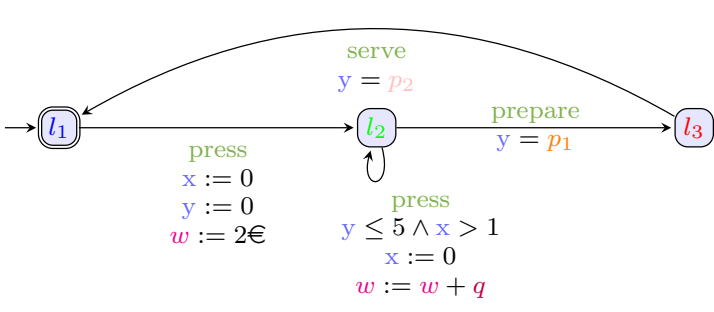

Figure 3: A PWTA modeling a coffee machine

- $e_{2}=\left\langle l_{2}, g, a, R, l_{2}\right\rangle$ where $g$ is $\mathrm{y} \leq 5 \wedge \mathrm{x}>1$ and $R$ sets $\mathrm{x}$ to $0, \alpha$ is $w:=w+q$,

- $e_{3}=\left\langle l_{2}, g, a, R, l_{3}\right\rangle$ where $g$ is $\mathrm{y}=p_{1}$ and

- $e_{4}=\left\langle l_{3}, g, a, R, l_{1}\right\rangle$ where $g$ is $\mathrm{y}=p_{2}$.

Let us now define the concrete semantics of PWTA as the union over all timing parameter and weight parameter valuations.

Definition 2 (Semantics of a valuated PWTA). Given a PWTA $\mathcal{A}=\left(\Sigma, L, l_{0}, F, \mathbb{C}, \mathbb{T} \mathbb{P}, \mathbb{W}, \mathbb{W} \mathbb{P}, I, E\right)$, a timing parameter valuation $t v$, and a weight parameter valuation $w v$, the semantics of $t v \mid w v(\mathcal{A})$ is given by the timed transition system (TTS) $\left(S, s_{0}, \rightarrow\right)$, with

- $S=\left\{(l, \nu, \mu) \in L \times \mathbb{R}_{+}^{H} \times \mathbb{Q}^{M} \mid \nu \models t v(I(l))\right\}$,

- $s_{0}=\left(l_{0}, \overrightarrow{0}_{\mathbb{C}}, \overrightarrow{0}_{\mathbb{W}}\right)$

- $\rightarrow$ consists of the discrete and (continuous) delay transition relations: $i)$ discrete transitions: $(l, \nu, \mu) \stackrel{e}{\mapsto}$ $\left(l^{\prime}, \nu^{\prime}, \mu^{\prime}\right)$, if $(l, \nu, \mu),\left(l^{\prime}, \nu^{\prime}, \mu^{\prime}\right) \in S$, and there exists $e=\left(l, g, a, R, \alpha, l^{\prime}\right) \in E$, such that $\nu \models t v(g)$, $\nu^{\prime}=[\nu]_{R}$, and $\mu^{\prime}=\operatorname{eval}_{w v}(\alpha, \mu)(w)$; ii) delay tran- sitions: $(l, \nu, \mu) \stackrel{d}{\mapsto}(l, \nu+d, \mu)$, with $d \in \mathbb{R}_{+}$, if $\forall d^{\prime} \in[0, d],\left(l, \nu+d^{\prime}, \mu\right) \in S$.

That is, a state is a triple made of the current location, the current (non-parametric) clock valuation, and the current (nonparametric) weight valuation. The clock valuations evolve naturally as in timed automata, while the current weight evolves according to the weight update function.

Moreover we write $(l, \nu, \mu) \stackrel{(e, d)}{\longrightarrow}\left(l^{\prime}, \nu^{\prime}, \mu^{\prime}\right)$ for a combination of a delay and discrete transition if $\exists \nu^{\prime \prime}:(l, \nu, \mu) \stackrel{d}{\mapsto}$ $\left(l, \nu^{\prime \prime}, \mu\right) \stackrel{e}{\mapsto}\left(l^{\prime}, \nu^{\prime}, \mu^{\prime}\right)$. Given $t v \mid w v(\mathcal{A})$ with concrete semantics $\left(S, s_{0}, \rightarrow\right)$, we refer to the states of $S$ as the concrete states of $t v \mid w v(\mathcal{A})$. A run of $t v \mid w v(\mathcal{A})$ is an alternating sequence of concrete states of $t v \mid w v(\mathcal{A})$ and pairs of edges and delays starting from the initial state $s_{0}$ of the form $s_{0},\left(e_{0}, d_{0}\right), s_{1}, \cdots$ with $i=0,1, \ldots, e_{i} \in E, d_{i} \in \mathbb{R}_{+}$and $\left(s_{i}, e_{i}, s_{i+1}\right) \in \rightarrow$.

Example 2. A concrete execution of the PWTA $t v \mid w v(\mathcal{A})$ of Example 1 with $w=2 €, w v(q)=0.5 €, t v\left(p_{1}\right)=5$ and $t v\left(p_{2}\right)=8$ is

$$
\begin{aligned}
& \left.\left(l_{1},(0,0),(0)\right) \quad \stackrel{\text { press, }}{\longrightarrow}\right) \quad\left(l_{2},(0,0),(2)\right) \quad \stackrel{\text { (press, } 1.5)}{\longrightarrow} \\
& \left(l_{2},(0,1.5),(2.5)\right) \stackrel{\text { (press,1) }}{\longrightarrow} \quad\left(l_{2},(0,2.5),(3)\right) \quad \stackrel{\text { (prepare, } 2.5)}{\longrightarrow} \\
& \left(l_{3},(2.5,5),(3)\right) \stackrel{\text { (serve, } 3)}{\longrightarrow}\left(l_{1},(5.5,8),(2.5)\right) \text {. }
\end{aligned}
$$

\section{TRAnslation of AFTs to PTAs}

\section{A. Overview of the translation}

We will model an attack-fault tree using a network of PWTAs that will synchronize along actions (using the usual composition semantics). Each gate and each leaf (i. e., BAS or BCF) will be modeled as a PWTA. Leaves PWTA have a duration and a weight, while gates PWTA store the weight value of their children to forward it to their parents. Therefore, 


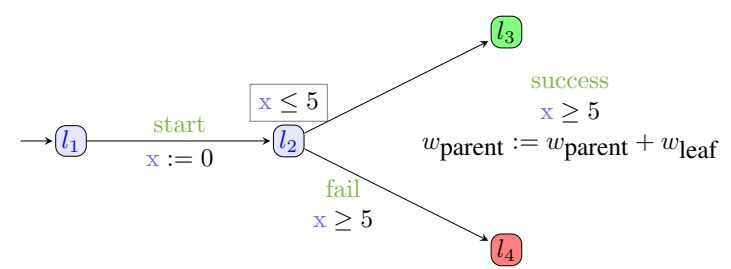

Figure 4: PWTA translation of leaf that can reach the success location in exactly 5 units of time, and of weight $w_{\text {leaf }}$

each gate PWTA maintains its own weight, and its value will be added to that of their parents in case of success (thanks to the parametric weight update).

All gates and leaves PWTAs initially synchronize their start action-referred as activation in this paper-, and end with either a success or fail synchronization action. After gates synchronize their start action, they synchronize the start action of their children

Intuitively, the process is top-bottom-top: the top level gate PWTA activates its children, which themselves activate their children (if any), and so on until the leaves PWTAs at the bottom of the attack-falt tree. Once a leaf PWTA terminates, it synchronizes either its success or fail action. In case of success, the leaf PWTA forwards its weight value to its parent, where this value is stored. When its parent gate PWTA terminates, the gate PWTA synchronizes either a success or a fail action. In case of success, the gate PWTA forwards its weight value to its parent, and so on until the top-level gate PWTA terminates.

If the top-level PWTA terminates in its success location, the attack is successful. We apply the reachability synthesis algorithm of PTAs on the success location in the top-level PWTA, that is, we synthesize all valuations for which this location is reachable: this gives us the success conditions of an attack. The set of constraints on time and weight that allowed this attack to be successful are output by this analysis.

As a running example, we consider the attack tree in Fig. 2 taken from [23].

\section{B. Translation of leaves}

A BAS/BCF is modeled as a PWTA with clocks and weights (see Fig. 4). Note that in real life while a BAS needs to success so the attack is possibly successful, a BCF needs to fail in order to propagate a disruption (as in basic component failure). However we consider in our models that both BAS and BCF need to reach the success location. There is two paths in a BAS/BCF PWTA, one that reaches the success location and one that reaches the fail location. In case of success, its weight is forwarded to and stored in its parent gate.

\section{Translation of gates}

Concrete translations of SAND, AND, OR gates are given in Table I (yellow locations denote urgency: time cannot elapse). Other gates are similar. a) AND: recall that an AND gate is disrupted if all of its children are disrupted. It activates all of its children then waits for their disruptions regardless of the order of the successes. At any moment if one fails, the AND gate fails. If the success action is synchronized, its parent weight $w_{\text {parent }}$ is updated: the weight $w_{\text {AND }}$ carried by the AND gate is added to $w_{\text {parent }}$.

Example 3. We give in Fig. 5b the PWTA corresponding to the AND gate access_home_network of Fig. 2. When all children are activated in the PWTA of Fig. 5b, there are four paths leading to the fail state, while only two (success of the two children in any order) leading to the success state. startAND3 launches the AND gate access_home_network. Both children, the BAS get_credential and the OR gate gain_access_to_private_networks are activated with the synchronization of the actions launchGetCred and startOR. Unlike the SAND gate, AND gate waits for any of its child to synchronize a success action. If successGetCred is synchronized, it then will wait for success $O R$ to go to the location success. If failGetCred is synchronized, the automaton will go to the location failing. When waiting for the action success $O R$, if failOR is synchronized the automaton will also go to the location failing. The other possibility (successOR then successGetCred) is similar. When in location failing it synchronizes the action failAND3, while if going to the location success it will synchronize the action successAND3. If the success state is reached, the weight of its parent gate is increased by its own weight.

b) SAND: recall that a SAND gate is disrupted if all its children from left to right are disrupted sequentially from left to right. It activates its leftmost child then waits for its success or failure, then activates its second leftmost child and so on. If the rightmost child succeeds, the SAND gate is disrupted. If one child fails, the SAND gate fails. For a SAND gate modeled as a PWTA with $n$ children, there is only one path leading to the success state, while there are $n$ paths leading to the fail state (one from each child). If the success action is synchronized, its parent weight $w_{\text {parent }}$ is updated: the weight $w_{\text {SAND }}$ carried by the SAND gate is added to $w_{\text {parent }}$.

Example 4. The top event of the attack tree in Fig. 2 is a SAND gate. We give the PWTA corresponding to this SAND in Fig. 5a. It synchronizes the action startSAND. Then it activates its leftmost child access_home_network with the action startAND3, which is an AND gate. If the action successAND3 is synchronized, its second leftmost child is activated with the action launchExploit. If the action successExploit is synchronized, its third and last child is activated with the action launchRunMScript. If the action successRunMScript is synchronized, the action successSAND is synchronized. At any moment, if one of its children fail and an action failAND3, failExploit or failRunMscript is synchronized the automaton goes to the location failing where the action failSAND is synchronized. If the success state is reached, the weight of its parent gate is increased by its own weight. 

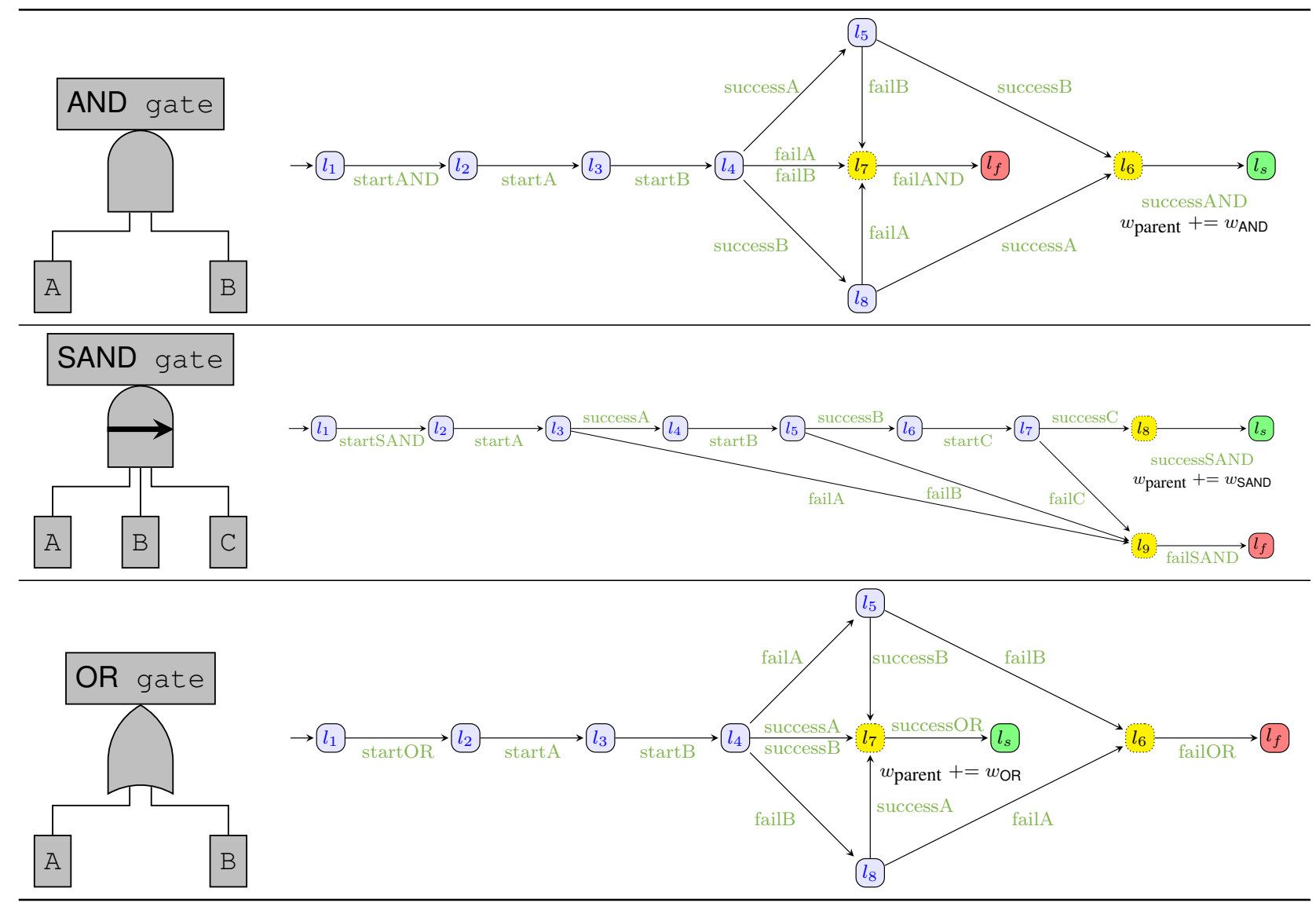

Table I: Translation rules of AND, SAND and OR gates to PWTA

c) OR: OR gate initially activates all of its children. OR gate is disrupted if at least one of its children is disrupted, and fails if all of its children fail. Therefore in the case of two children, one child can fail and the OR gate still propagates a disruption if the other one succeeds right after. However, if one child succeeds no need to wait for the second one and the success action of the OR gate is synchronized. If the success action is synchronized, its parent weight $w_{\text {parent }}$ is updated: the weight $w_{\mathrm{OR}}$ carried by the OR gate is added to $w_{\text {parent }}$.

\section{Top-level automaton}

Finally, we need to create an automaton that will activate the first top-event gate of the AFT. We call it rootTA. This PWTA is the one that starts the chain reaction by activating the top-event PWTA gate, which at its turn will activate its own children and so on. It waits for the success or fail action of this PWTA gate. In case of success, its weight has been updated with the total weight value of the execution forwarded by the top-event gate PWTA. This bottom-to-top addition stores in the weight current_cost_root the total weight of the attack. The rootTA also stores the total time spent since the first activation of the top-event PWTA (using an extra clock and parameter).
Example 5. We give in Fig. 6 the top-level PWTA for the AFT in Fig. 2. It is very similar to a leaf PWTA. It activates the toplevel gate PWTA, then waits for its success or fail action. If the success action is synchronized, its weight has been updated to the total weight value of the execution and is checked against an additional parameter total_cost so IMITATOR outputs this current_cost_root value. Likewise, the clock abs_time which is never reset since the activation of rootTA is checked against a timing parameter total_time. Therefore IMITATOR outputs the total time of execution.

\section{IMPLEMENTATION OF THE TRANSLATION}

\section{A. IMITATOR}

IMITATOR [15] is a parametric model checker taking as input an extension of networks parametric timed automata extended with synchronization, stopwatches and discrete variables. IMITATOR supports global (shared) discrete rationalvalued variables, that can be either concrete (in which case they are syntactic sugar for an unbounded number of locations), or symbolic, in which case they can be updated to or compared with parameters. While IMITATOR technically considers a single type of parameters (where symbolic variables can be compared or even updated to timing parameters), our 

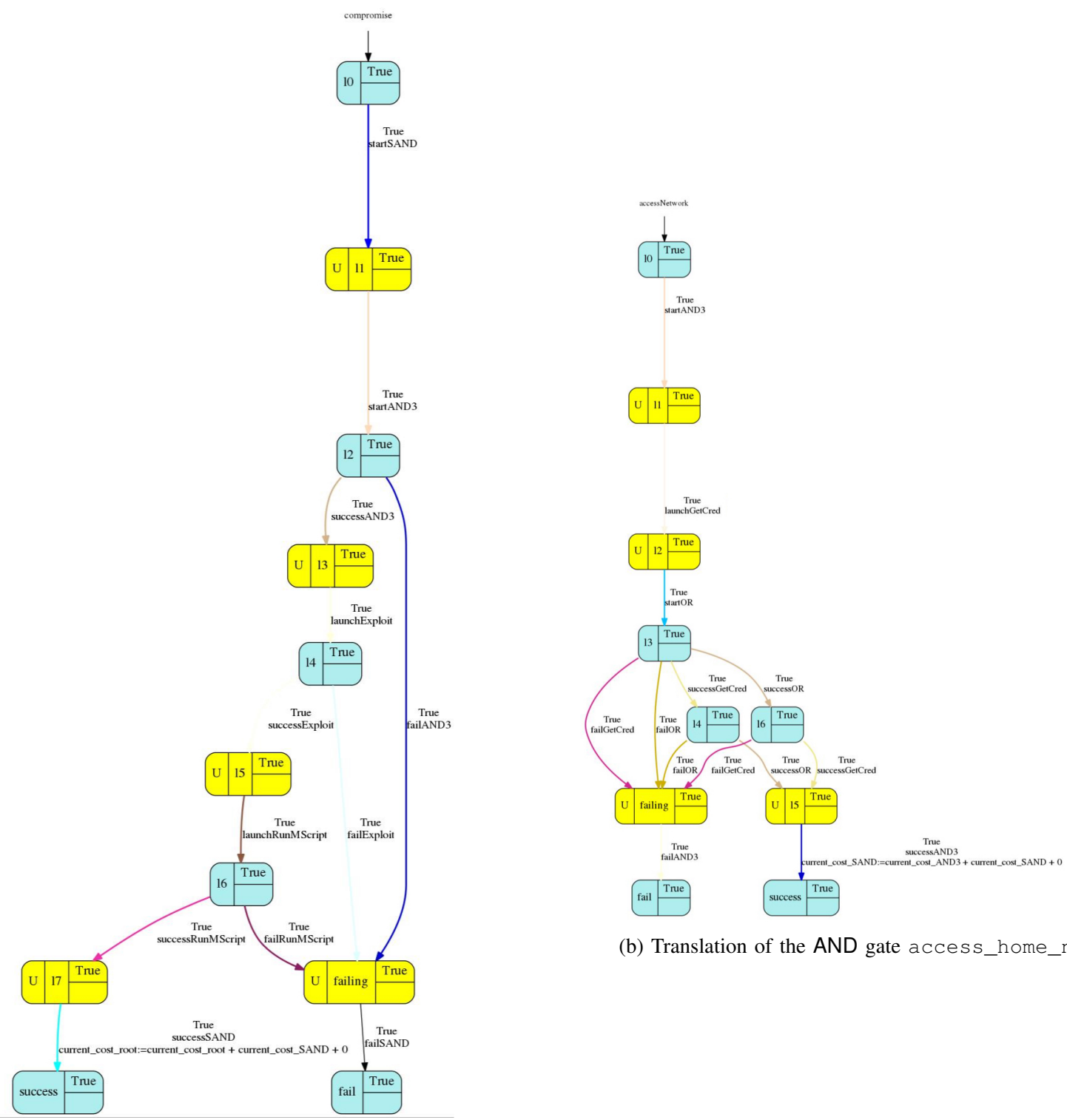

(b) Translation of the AND gate access_home_network

(a) Translation of the top-level SAND gate

Figure 5: SAND and AND gate

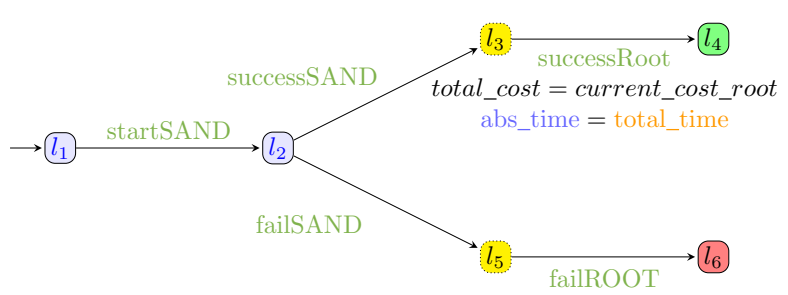

Figure 6: The rootTA weight parameters are never compared to timing parameters, and this setting can be considered as a subclass of the IMITATOR expressiveness.

IMITATOR implements several synthesis algorithms, notably reachability synthesis (EFsynth), that attempts to synthesize all parameter valuations for which a given location is reachable-which is the algorithm we use here. IMITATOR relies on the symbolic semantics of parametric timed automata (see e.g., [32], [33]), where symbolic states are made of a discrete location, and a constraint over the clocks and parameters. The weight parameters are added to this symbolic 
semantics in a straightforward manner, with symbolic states enriched with linear constraints over weight parameters.

Note that, while parametric timed automata are highly undecidable (see [34] for a survey), and while our parametric extension adds a new layer of complexity, all analyses terminate with an exact result (sound and complete) because our models are acyclic: our AFTs are trees, and their translation yields structurally acyclic PWTAs. As a consequence, the symbolic semantics of these PWTAs can be represented as a finite structure, and the analysis is guaranteed to terminate.

\section{B. Translation from AFTs to PWTAs}

The translation from AFTs to PWTAs was implemented within the framework of ATTop [23]. The existing software ATTop can take as input a Galileo formatted file. The code in Fig. 7 expresses an attack-fault tree of one OR gate named A, with two children $B$ and $C$. The BAS B takes between 50 and 100 units of time to terminate, and costs $\$ 50$ to the attacker. The BAS $\mathrm{C}$ takes between 30 and 70 units of time to terminate, and costs $\$ 30$ to the attacker.

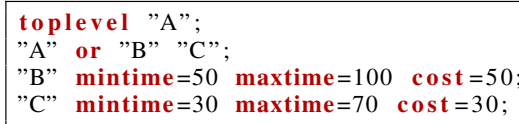

Figure 7: Example of Galileo attack-tree

ATTop takes as an input a Galileo file and parses it to represent it as an attack-fault tree meta-model (ATMM) (see [23, Section 3] for a screenshot of the tool). In our approach we directly translate the representation of the ATMM into an IMITATOR formatted file, using the Epsilon Generation Language (EGL) [35]. This translation is a very efficient way to obtain AFTs modeled using PWTAs: designing manually a PWTA model from an AFT is very tedious to achieve, while defining an AFT within the Galileo syntax is simple.

Once the PWTA obtained, we synthesize using IMITATOR all parameter valuations for which the success location of the rootTA can be reached (using EFsynth). These sets of parameter values will help us to determine attack and fault scenarios in the following section.

\section{CASE STUdies}

As a proof of concept, we apply our approach to an attack tree from the literature and an original attack-fault tree. Experiments were conducted with IMITATOR 2.10.4 "Butter Jellyfish", 4 on a $2.4 \mathrm{GHz}$ Intel Core i5 processor with $2 \mathrm{GiB}$ of RAM in a VirtualBox environment. Computation times of parameter values ranges from 1 to 9 seconds with four parameters.

${ }^{4}$ Sources, binaries, models and results are available at https://www.imitator. fr/static/ACSD19PAT/

\section{A. Compromising an IoT device}

We apply our approach to the AFT depicted in Fig. 2. We choose to parametrize the cost of finding a LAN access point (CostFindLAN_AP) and the maximum amount of time to break WPA keys (tMax_Break) of the AFT. This configuration will describe which attack (WLAN or LAN) is smarter for the attacker, depending on their resources: finding a LAN access point can be difficult depending on the infrastructure security and perhaps social engineering is needed. However, if the attacker does not have enough resources but a large amount of time (s)he can spend time trying to break WPA keys. IMITATOR computes several constraints on these parameters such that the attack is successful.

Different constraints are possible representing possible time and weight values s.t. an attack is possible. This is represented as a disjunction of conjunctions of constraints on parameters. For instance it can be a quick but very costly attack, or a long but cheap one; therefore different attack and fault scenarios appear. The conjunction of constraints

$$
\begin{gathered}
2 * \text { tMax_Break } \geq 23 \wedge \text { CostFindLAN_AP } \geq 0 \\
\wedge \text { CostFindLAN_AP }+180=\text { total_cost } \wedge 2 * \text { total_time }=23
\end{gathered}
$$

represents an attack that is very expensive for the attacker: indeed, the total cost of the attack is at least $\$ 180$ and fully depends on the cost of finding a LAN access point. However, the time spent on the attack is negligible and fixed (11.5h).

In opposition, the constraint

$$
\begin{gathered}
2 * \text { tMax_Break }+3 \geq 2 * \text { total_time } \\
\wedge \text { CostFindLAN_AP } \geq 0 \\
\wedge 2 * \text { total_time } \geq 23 \wedge \text { total_cost }=232
\end{gathered}
$$

shows a an attack that will last at least $11.5 \mathrm{~h}$ - that is, the attacker does not exactly knows when (s)he will break the WPA keys depending for instance of her/his computation power-but with a fixed cost of $\$ 232$.

Contrarily to our initial intuition, the cost of this second attack can be high above the first one, as breaking the WPA keys is quite costly (\$80) in opposition with finding a LAN access point. A smart attacker could choose, regardless of their time and resources the first attack through LAN access point.

\section{B. SpaceX rocket Falcon 9 explosion}

Our second case study is an adaptation of the anomaly investigation that followed the explosion of SpaceX rocket Falcon 9 in september $2016^{5}$. The AFT in Fig. 8 depicts the different configurations that can eventually end up with the explosion. The objective of this case-study is to show that the explosion is more likely to be accidental, due to the expensiveness of the BAS for the attacker who could attempt a sabotage.

${ }^{5}$ SpaceX anomaly update, https://www.spacex.com/news/2016/09/01/ anomaly-updates 


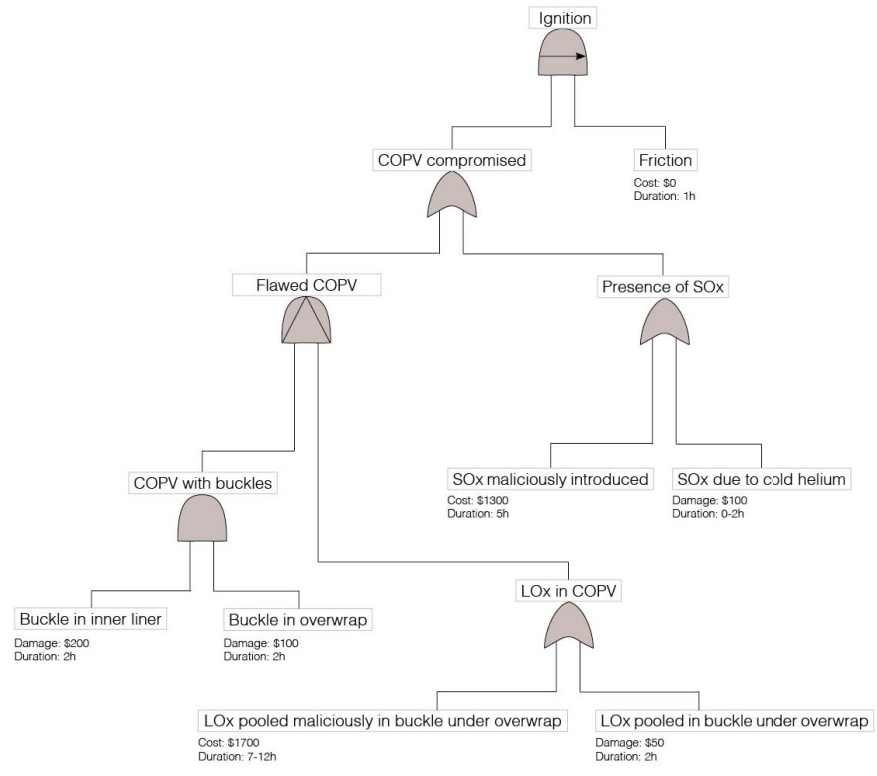

Figure 8: AFT of SpaceX rocket explosion

The rocket carries a helium tank with three composite overwrapped pressure vessels (COPVs) inside. One COPV possibly had a manufacturing defect and buckles in its liner and the carbon overwrap (AND gate). Afterwards (PAND gate) liquid oxygen (LOx) can pool in these buckles and become trapped when pressurized under the carbon overwrap, resulting in a flawed COPV. An other possibility is the presence of solid oxygen ( $\mathrm{SOx}$ ) either due to the loading temperature of helium or placed here intentionally by an attacker (OR gate).

These two configurations result in a compromised COPV. When the COPV is compromised, a friction due to take-off tests can start the rocket ignition (SAND gate).

$\mathrm{BCF}$ have a duration representing the time taken until the component failure. Damage is the cost for the organization for having built a defective component, or the cost induced when the component has failed. BASs have a cost for the attacker to perform the attack, and a duration for the attack to be successful.

We choose to parametrize the damages induced to the manufacturing facility by damage_BuckleInInnerLiner, and the cost of pooling solid oxygen near the COPV, cost_SOXmaliciouslyIntroduced.

The constraint

$$
\begin{gathered}
13 \geq \text { total_time } \geq 8 \\
\text { ^cost_SOXmaliciouslyIntroduced } \geq 0 \\
\wedge \text { total_damages } \geq 100 \\
\text { ^damage_BuckleInInnerLiner }+100=\text { total_damages } \\
\wedge \text { total_cost }=1700
\end{gathered}
$$

represents the attack using the malicious introduction of LOx between the inner liner and the carbon overwrap of the COPV.
Clearly this attack is very costly (\$1700) and assumes the presence of these buckles. It is highly prejudicial to SpaceX as the company may want to investigate the manufacturing facility that produces COPV components.

The other attack, represented by the constraint

$$
\begin{gathered}
\text { total_time }=6 \\
\wedge \text { cost_SOXmaliciouslyIntroduced } \geq 0 \\
\wedge \text { total_damages }=0 \\
\wedge \text { damage_BuckleInInnerLiner } \geq 0 \\
\wedge \text { total_cost }=\text { cost_SOXmaliciouslyIntroduced }
\end{gathered}
$$

shows that the cost of the attack is equal to the cost of introducing SOx near the COPV. The higher is the parameter cost_SOXmaliciouslyIntroduced, the higher is the cost of the attack. We may assume this cost is high enough as SpaceX surely secured its launch complex. Otherwise, an efficient counter-measure would be to find means to increase this cost for the attacker.

The constraint

$$
\begin{gathered}
\text { cost_SOXmaliciouslyIntroduced } \geq 0 \\
\wedge \text { total_damage } \geq 150 \\
\wedge \text { damage_BuckleInInnerLiner }+150 \geq \text { total_damage } \\
\wedge \text { total_time }=3 \wedge \text { total_cost }=0
\end{gathered}
$$

represents the fact that buckles in the inner liner and in the carbon overwrap of the COPV, and then LOx pooled under the overwrap, lead to a complete failure of the system, i.e., the rocket explodes. In this scenario, there is in all likelihood no attacker. However, the damages for the manufacturing facility can be huge if it is flawed: SpaceX should probably investigate in their manufacturing facilities in order to prevent the production of other flawed components.

Finally, the constraint

$$
\begin{gathered}
\text { cost_SOXmaliciouslyIntroduced } \geq 0 \\
\wedge \text { total_damage }=100 \\
\wedge \text { damage_BuckleInInnerLiner } \geq 0 \\
\wedge 3 \geq \text { total_time } \geq 1 \wedge \text { total_cost }=0
\end{gathered}
$$

shows that the explosion can be provoked by the presence of SOx due to cold helium. This case is possible without any attacker or component failure and is therefore fully accidental. No damages are caused to SpaceX (excepted the cost of the unusable rocket) or its suppliers.

These scenarios indicate that the rocket explosion is more likely to be accidental, as the cost in both scenarios where there is an attacker is very high. However, the worst case indicates that SpaceX should investigate their production lines to prevent other flawed components, as well as the presence of an attacker.

\section{CONCLUSION}

We addressed the problem of formalizing attack-fault trees in a more abstract framework allowing to cope with parametric 
timings, costs and damages. We defined and implemented a translation from attack-fault trees to PWTAs (a new extension of PTAs) that can be analyzed using the IMITATOR modelchecker. This translation allows us to define easily an AFT using the Galileo syntax, and obtain as an output this AFT modeled with PWTAs. Using IMITATOR, we synthesize all parameter values such that there is a successful attack and/or a system failure. Finally, obtaining a disjunction of convex sets of parameter values allows us to define different attack and fault scenarios. Therefore it helps selecting the most plausible scenario and the most efficient counter-measures.

Future works: In this paper, we only considered three parameters: timing, cost and damage parameters. However, it is trivial to split these parameters into more precise ones, such as human damages (health and insurance) and material damages caused by the attacker or the failure of the system: an attack can be cheap for the attacker but inflict many kind of damages to the organization, as in our SpaceX case study. Thanks to the vector of weights defined in our PWTAs, this would be immediate to consider in our framework and implementation.

Moreover, extending our framework to attack-defense trees [9], [36] is also on our agenda.

Finally, adding probabilities in order to create probabilistic parametric attack-fault trees will be an interesting and challenging future work. Indeed, in our SpaceX rocket case study adding probabilities to the manufacturing defects of the COPV on top of the damages inflicted to the company would strengthen considerably our formalism.

\section{ACKNOWLEDGEMENTS}

We are thankful to Stefano Schivo and Enno J.J. Ruijters from Twente University for their helpful advices concerning meta-models and ATTop.

\section{REFERENCES}

[1] NATO Research and Technology Organisation, "Improving common security risk analysis," Tech. Rep. AC/323(ISP-049)TP/193, 2008.

[2] "OWASP. CISO AppSec guide: Criteria for managing application security risks," 2013.

[3] C. Salter, O. S. Saydjari, B. Schneier, and J. Wallner, "Toward a secure system engineering methodology," in NSPW, 1998, pp. 2-10.

[4] B. Kordy, S. Mauw, S. Radomirovic, and P. Schweitzer, "Foundations of attack-defense trees," in FAST, ser. LNCS, vol. 6561. Springer, 2010, pp. 80-95.

[5] B. Kordy, L. Piètre-Cambacédès, and P. Schweitzer, "Dag-based attack and defense modeling: Don't miss the forest for the attack trees," Computer Science Review, vol. 13-14, pp. 1-38, 2014.

[6] I. N. Fovino, M. Masera, and A. D. Cian, "Integrating cyber attacks within fault trees," RESS, vol. 94, no. 9, pp. 1394-1402, 2009.

[7] E. Ruijters and M. Stoelinga, "Fault tree analysis: A survey of the stateof-the-art in modeling, analysis and tools," Computer Science Review, vol. 15, pp. 29-62, 2015.

[8] A. Bagnato, B. Kordy, P. H. Meland, and P. Schweitzer, "Attribute decoration of attack-defense trees," IJSSE, vol. 3, no. 2, pp. 1-35, 2012.

[9] B. Kordy, S. Mauw, S. Radomirovic, and P. Schweitzer, "Attack-defense trees," JLC, vol. 24, no. 1, pp. 55-87, 2014.

[10] R. Kumar and M. Stoelinga, "Quantitative security and safety analysis with attack-fault trees," in HASE. IEEE, 2017, pp. 25-32.

[11] R. Alur, T. A. Henzinger, and M. Y. Vardi, "Parametric real-time reasoning," in STOC. New York, NY, USA: ACM, 1993, pp. 592601 .
[12] G. Behrmann, A. Fehnker, T. Hune, K. G. Larsen, P. Pettersson, J. Romijn, and F. W. Vaandrager, "Minimum-cost reachability for priced timed automata," in HSCC, ser. LNCS, vol. 2034. Springer, 2001, pp. $147-161$.

[13] R. Alur, S. La Torre, and G. J. Pappas, "Optimal paths in weighted timed automata," TCS, vol. 318, no. 3, pp. 297-322, 2004.

[14] R. Kumar, S. Schivo, E. Ruijters, B. M. Yildiz, D. Huistra, J. Brandt, A. Rensink, and M. Stoelinga, "Effective analysis of attack trees: A model-driven approach," in FASE, ser. LNCS, vol. 10802. Springer, 2018, pp. 56-73

[15] É. André, L. Fribourg, U. Kühne, and R. Soulat, "IMITATOR 2.5: A tool for analyzing robustness in scheduling problems," in FM, ser. LNCS, vol. 7436. Springer, 2012, pp. 33-36.

[16] R. Kumar, E. Ruijters, and M. Stoelinga, "Quantitative attack tree analysis via priced timed automata," in FORMATS, ser. LNCS, vol. 9268. Springer, 2015, pp. 156-171.

[17] R. Kumar, D. Guck, and M. Stoelinga, "Time dependent analysis with dynamic counter measure trees," CoRR, vol. abs/1510.00050, 2015.

[18] F. Arnold, D. Guck, R. Kumar, and M. Stoelinga, "Sequential and parallel attack tree modelling," in SAFECOMP Workshops, ser. LNCS, vol. 9338. Springer, 2015, pp. 291-299.

[19] M. Gribaudo, M. Iacono, and S. Marrone, "Exploiting bayesian networks for the analysis of combined attack trees," ENTCS, vol. 310, pp. 91-111, 2015.

[20] G. Dalton, R. Mills, J. Colombi, and R. Raines, "Analyzing attack trees using generalized stochastic Petri nets," 2006, pp. 116 - 123.

[21] Z. Aslanyan, F. Nielson, and D. Parker, "Quantitative verification and synthesis of attack-defence scenarios," in CSF. IEEE Computer Society, 2016, pp. 105-119.

[22] H. Hermanns, J. Krämer, J. Krcál, and M. Stoelinga, "The value of attack-defence diagrams," in POST, ser. LNCS, vol. 9635. Springer, 2016, pp. 163-185.

[23] S. Schivo, B. M. Yildiz, E. Ruijters, C. Gerking, R. Kumar, S. Dziwok, A. Rensink, and M. Stoelinga, "How to efficiently build a front-end tool for UPPAAL: A model-driven approach," in SETTA, ser. LNCS, vol. 10606. Springer, 2017, pp. 319-336.

[24] M. Hendriks and M. Verhoef, "Timed automata based analysis of embedded system architectures," in IPDPS. IEEE, 2006.

[25] A. Remke and M. Stoelinga, Eds., Proceedings of the International Autumn School on Stochastic Model Checking. Rigorous Dependability Analysis Using Model Checking Techniques for Stochastic Systems (ROCKS 2012), ser. LNCS, vol. 8453. Springer, 2014.

[26] A. David, K. G. Larsen, A. Legay, M. Mikucionis, and D. B. Poulsen, "Uppaal SMC tutorial," STTT, vol. 17, no. 4, pp. 397-415, 2015.

[27] A. David, K. G. Larsen, A. Legay, M. Mikucionis, D. B. Poulsen, J. van Vliet, and Z. Wang, "Statistical model checking for networks of priced timed automata," in FORMATS, ser. LNCS, vol. 6919. Springer, 2011, pp. 80-96.

[28] B. Kordy and W. Widel, "On quantitative analysis of attack-defense trees with repeated labels," in POST, ser. LNCS, vol. 10804. Springer, 2018, pp. 325-346.

[29] R. Chevallier, E. Encrenaz-Tiphène, L. Fribourg, and W. Xu, "Timed verification of the generic architecture of a memory circuit using parametric timed automata," $F M S D$, vol. 34, no. 1, pp. 59-81, 2009.

[30] R. Alur and D. L. Dill, "A theory of timed automata," TCS, vol. 126 , no. 2, pp. 183-235, 1994.

[31] F. Wang, "Parametric analysis of computer systems," FMSD, vol. 17, no. 1, pp. 39-60, 2000.

[32] É. André, Th. Chatain, E. Encrenaz, and L. Fribourg, "An inverse method for parametric timed automata," International Journal of Foundations of Computer Science, vol. 20, no. 5, pp. 819-836, 2009.

[33] A. Jovanović, D. Lime, and O. H. Roux, "Integer parameter synthesis for timed automata," TSE, vol. 41, no. 5, pp. 445-461, 2015.

[34] É. André, "What's decidable about parametric timed automata?" STTT, 2019, to appear.

[35] L. M. Rose, R. F. Paige, D. S. Kolovos, and F. Polack, "The epsilon generation language," in ECMDA-FA, ser. LNCS, vol. 5095. Springer, 2008, pp. 1-16.

[36] O. Gadyatskaya, R. R. Hansen, K. G. Larsen, A. Legay, M. C. Olesen, and D. B. Poulsen, "Modelling attack-defense trees using timed automata," in FORMATS, ser. LNCS, vol. 9884. Springer, 2016, pp. $35-50$. 